\title{
Avaliação do efeito da quercetina em ratos Wistar com Síndrome Metabólica
}

\section{Hevista}

Evaluation of the effect of quercetin in Wistar rats with

metabolic syndrome

(1) Marcella Martins Terra ${ }^{1}$, Hussen Machado ${ }^{1}$, Hélady Sanders Pinheiro ${ }^{1}$, Martha de Oliveira Guerra ${ }^{1}$, Vera M Peters ${ }^{1}$

${ }^{1}$ Centro de Biologia da Reprodução, Universidade Federal de Juiz de Fora, Juiz de Fora, MG.

\section{Marcella Terra}

Centro de Biologia da Reprodução - CBR/Universidade Federal de Juiz de Fora - UFJF

Rua José Lourenço Kelmer, s/n, Campus Universitário Bairro: São Pedro.

CEP: $36036-000$

Juiz de Fora - MG

刃 marcellaterra@yahoo.com.br

\section{RESUMO}

Introdução: O tratamento da síndrome metabólica (SM) é um desafio, uma vez que terapias não medicamentosas são de difícil implementação e o tratamento farmacológico ideal não está totalmente estabelecido. Objetivo: Avaliar o efeito da quercetina na pressão arterial (PA), dislipidemia e acúmulo de gordura visceral em modelo experimental de SM induzida por dieta hiperlipídica. Material e Métodos: Ratos Wistar receberam ração hiperlipídica a partir da quarta semana de vida, por 20 semanas. O grupo tratado recebeu quercetina a partir da oitava semana de vida. Avaliou-se semanalmente o peso corporal e a PA de cauda por pletismografia. Ao final do experimento foram realizados testes de perfil glicêmico e lipídico. Resultados: A administração de dieta hiperlipídica se associou ao desenvolvimento de SM, caracterizada por acúmulo central de gordura, hipertensão arterial, hiperglicemia e hipertrigliceridemia. A quercetina não apresentou eficácia no tratamento das comorbidades que compõem a SM. Conclusão: A administração crônica diária da quercetina em modelo experimental de SM induzida por dieta hiperlipídica não alterou de forma significante o perfil nutricional, metabólico e pressórico dos animais.

Palavras-chave: síndrome metabólica, quercetina, ratos wistar

\section{ABSTRACT}

Introduction: The treatment of the metabolic syndrome (MetS) is a challenge, since nonpharmacologic therapies are difficult to implement and the ideal pharmacologic treatment has not been completely established. Aim: To evaluate the effect of quercetin in blood pressure (BP), dyslipidemia, visceral fat accumulation, in an experimental model of MetS induced by a hyperlipidic diet. Material and Methods: Wistar rats received high fat diet feed from the fourth week of life for 20 weeks. The treatment group received quercetin from the eighth week of life. Body weight and tail BP through pletysmography were evaluated weekly. At the end of the experiment, tests of glucose and lipid profile. Results: The administration of a high fat diet was associated to the development of MetS, characterized by an accumulation of central fat, arterial hypertension, hyperglycemia, and hypertriglyceridemia. Quercetin was not effective in the treatment of comorbidities associated with MetS. Conclusion: Chronic daily administration of quercetin in an experimental model of MetS induced by a hyperlipidic diet did not significantly alter the nutritional, metabolic, and pressure profile of the animals.

Keywords: metabolic syndrome, quercetin, wistar rats 


\section{INTRODUÇÃO}

A síndrome metabólica (SM) é uma desordem multifatorial de alta prevalência que se manifesta por anormalidades fisiológicas e bioquímicas, caracterizada por resistência à insulina, obesidade visceral, elevação da pressão arterial e dislipidemia aterogênica (elevação dos triglicérides e redução nos níveis de lipoproteínas de alta densidade-colesterol) como também outras anormalidades como, disfunção endotelial e doenças cardiovasculares (HEART et al., 2004; SIMMONS et al., 2010).

A obesidade decorrente da ingestão de dietas hipercalóricas contribui para o desenvolvimento da SM e sabe-se que a obesidade está aumentando. Em todo o mundo existem cerca de dois bilhões de adultos com sobrepeso dos quais $39 \%$ são homens e $40 \%$ mulheres. Enquanto que, aproximadamente, um milhão de adultos são obesos $11 \%$ homens e $15 \%$ mulheres (WHO,2014). Sobrepeso e obesidade têm demonstrado aumentar com o passar das décadas, tornando-se um dos mais preocupantes problemas de saúde pública, pois pelo menos 2,8 milhões de pessoas morrem todo ano como resultado do sobrepeso ou obesidade (ENES; SLATER, 2010; GOULARTE et al., 2017; LIN et al.., 2017).

Estudos epidemiológicos e clínicos têm confirmado que o sobrepeso está relacionado ao aumento da morbidade e mortalidade em virtude de sua frequente associação com outras doenças que compõem a síndrome metabólica (SM) e, devido às patologias integrantes da SM o paciente estaria sob maior risco (SIMMONS et al., 2010).

A gordura visceral é a principal contribuinte para as alterações metabólicas devido à produção de adipocinas, que são citocinas presentes nos adipócitos, além disso, existe associação direta da obesidade com a atividade simpática e com a inflamação. Essa constatação desencadeou grande preocupação em combater a obesidade, que é um fator de risco, per se, e que de certa forma contribui para a emergência de outros riscos (GÓMEZ-HERNÁNDEZ et al., 2016). Em todo o mundo, 2,8 milhões de pessoas morrem a cada ano como resultado de estar com sobrepeso ou obesos (NG et al., 2014). Pelo exposto, o controle da obesidade e a minimização dos efeitos associados à SM são necessários e diversos fármacos vêm sendo pesquisados para o seu tratamento.

Estudos sugerem que dietas ricas em flavonóides têm a propriedade de reduzir o risco de doenças degenerativas, tais como os eventos coronários, as doenças cardiovasculares, a dislipidemia e o diabetes (PEREZ-VIZCAINO et al., 2009). Os flavonóides, especialmente a quercetina, estão amplamente distribuídos nas plantas e encontram-se presentes em quantidades consideráveis em frutas e vegetais (BIRT; HENDRICH; WANG, 2001). A quercetina $\left(3,5,7,3^{\prime}, 4^{\prime}\right.$-pentahidroxiflavona) possui propriedades antioxidantes, antinflamatórias e vasodilatadoras, que em estudos em animais (PEREZ-VIZCAINO et al., 2009; YANG et al., 2018) e humanos (EDWARDS et al., 2007; OŻAROWSKI et al., 2018) parece eficaz no tratamento das comorbidades que compõem a SM.

A utilização de modelos experimentais de SM constitui uma alternativa para a avaliação de drogas anti-hipertensivas, hipoglicemiantes e hipocolesterolêmicas. Dentre estes modelos, os que são induzidos por modificações dietéticas são os que melhor reproduzem as alterações metabólicas decorrentes do acúmulo de tecido adiposo (RAMALHO; GUIMARÃES, 2008; SHEN et al., 2004). Para tanto utilizam-se ratos Wistar obesos, um modelo animal que apresenta muitas das características da síndrome metabólica humana, uma vez que estes animais exibem resistência à insulina, dislipidemia, hiperinsulinemia e hipertensão (BROWN; PANCHAL, 2011; MACHADO, H. et al., 2012).

No presente estudo avaliaram-se os efeitos da administração crônica diária da quercetina, sobre a pressão arterial, glicemia, dislipidemia e acúmulo de gordura visceral, em um modelo experimental de SM induzida por dieta hiperlipídica.

\section{MATERIAL E MÉTODOS}

Todos os procedimentos experimentais foram aprovados pelo Comitê de Ética em Experimentação Animal da Universidade Federal de Juiz de Fora (Protocolo no 001/2009).

\section{Animais e dieta}

Ratos Wistar machos com quatro semanas de vida, fornecidos pelo Centro de Biologia da Reprodução (CBR) da Universidade Federal de Juiz de Fora foram alocados, aleatoriamente, em três grupos de 12 animais. No grupo controle (C), os animais foram alimentados com dieta comercial Nuvital $R$ (Nuvilab, Colombo-PR, Brasil), considerada normocalórica (carboidrato 68\%, proteína $19 \%$, lipídios 3,5\%, fibras $4,5 \%$, vitaminas e minerais $5 \%, 3,70 \mathrm{Kcal} / 100 \mathrm{~g}$ ) durante vinte semanas. No grupo síndrome metabólica (SM) os animais foram alimentados, pelo mesmo tempo, com dieta hiperlipídica pré-fabricada pela empresa PragSoluções Comércio e Serviços Ltda, Jaú-SP, Brasil), contendo: carboidrato $35 \%$, proteína $19 \%$, lipídios $36,5 \%$, fibras $4,5 \%$, vitaminas e minerais $5 \%, 5,20 \mathrm{Kcal} / 100 \mathrm{~g}$. No grupo Síndrome metabólica/quercetina (SMQ) os animais receberam a mesma dieta do grupo SM e, a partir da oitava semana de vida receberam $10 \mathrm{mg} /$ $\mathrm{kg} /$ dia de quercetina (Sigma Aldrick, Germany) por gavagem durante quatro meses (Fig.1).

Os animais foram alojados em gaiolas de propileno 
(quatro animais por gaiola), com livre acesso a ração e água, mantidos em alojamentos com temperatura constante $\left(22 \pm 2{ }^{\circ} \mathrm{C}\right)$, umidade relativa do ar $(60 \pm 10)$ e ciclo claro-escuro de 12 horas, iniciando-se a fase clara às 7 horas da manhã.

\section{Parâmetros avaliados}

\section{Peso corporal}

Consideramos os animais com sobrepeso quando a diferença de peso entre os grupos com dieta hiperlipídica (SM e SMQ) foi significativamente maior $(p<0.05)$ em relação ao grupo controle (C)(DOURMASHKIN et al., 2005; NASCIMENTO et al., 2008).

Para o cálculo de consumo alimentar (g) e energético (Kcal/dia), os animais foram pesados e alocados individualmente em gaiolas metabólicas, com quantidades fixas de dieta, uma vez por semana, por um período de 24 horas.

\section{Obesidade visceral}

Mensurada pelo peso (g) do tecido adiposo retroperitoneal, epididimários direito e esquerdo, obtidos por lipectomia abdominal bilateral, realizada após eutanásia (NASCIMENTO et al., 2008).

\section{Pressão arterial}

A pressão arterial sistólica (PAS) foi avaliada semanalmente, utilizando o método não invasivo da pletismografia de cauda (Pletismógrafo LE5001; Panlab®, Barcelona, Espanha). Após précondicionamento na câmara de contenção, os animais foram pré-aquecidos a $35 \pm 20 \mathrm{C}$ por 5 minutos e foram obtidas dez leituras consecutivas. A medida individual da pressão arterial foi calculada pela média das leituras obtidas (MACHADO et al., 2012; MACHADO, HUSSEN; DE OLIVEIRA GUERRA; MARIA PETERS, 2010).

\section{Glicemia e Lipidemia}

Os níveis séricos de glicose, triglicérides e colesterol total foram avaliados vinte semanas após o início das dietas, o sangue foi coletado após anestesia com cetamina (90 mg/kg ip.) e xilazina $(10 \mathrm{mg} / \mathrm{kg}$ ip.) (König $S A \circledast$, Avellaneda, Argentina) por punção cardíaca. A mensuração foi realizada após 8 horas de jejum e utilizando kits comerciais (Labtest $®$, Lagoa Santa, Brasil).

\section{Eutanásia}

Na vigésima semana os animais foram eutanasiados com sobredose de anestesia com cetamina $(90 \mathrm{mg} / \mathrm{kg}$ ip.) e xilazina (10 mg/kg ip.) (König $S A ®$, Avellaneda, Argentina).

\section{Estatística}

Os grupos controle e SM foram comparados pelo teste de variância ANOVA bicaudal, seguida do teste de Dunnet $a=0.05$. O programa SPSS 15.0 foi usado em todas as análises (SPSS Inc., Chicago, IL, USA).

\section{RESULTADOS}

\section{Peso corporal e Obesidade Visceral}

O grupo C apresentou maior ingestão diária de ração e água, quando comparado aos grupos SM e SMQ, entretanto a ingestão energética foi semelhante entre os grupos. O aumento no peso corporal dos grupos SM e SMQ foi acompanhado por maior acúmulo de tecido adiposo retroperitoneal e epididimário, quando comparado ao grupo $C$. Não foi observada diferença significativa no acúmulo de tecido adiposo quando os grupos SM e SMQ foram comparados $(p=0,4)$, tabela 1 .

Os animais dos grupos C, SM e SMQ apresentavam pesos semelhantes no início do experimento. Ao longo das 20 semanas de estudo, todos os animais, independentes do tipo de dieta ganharam peso. Porém na $10^{a}$ semana, os animais pertencentes aos grupos SM e SMQ apresentaram ganho de peso corporal de $17,5 \%$ e $13,7 \%$ superior ao grupo $C(p=0,004 ; p=0,011)$. Ao final do experimento essa porcentagem atingiu $29,8 \%$ e $27,5 \%$ superior ao grupo C. Durante todo o experimento não foram observada diferenças significantes no peso corporal do grupo SM em relação ao grupo SMQ.

\section{Pressão arterial}

A partir da $17^{a}$ semana de experimentação o grupo SM apresentou elevação dos níveis pressóricos (gráfico 1). Esse aumento foi confirmado na $20^{\mathrm{a}}$ semana, onde o grupo SM $(159 \pm 10,1 \mathrm{mmHg})$ e SMQ $(161 \pm 11,8 \mathrm{mmHg})$ apresentaram aumento significante em relação ao grupo C $(118 \pm 8,4 \mathrm{mmHg}, \mathrm{p}<0,001)$.

\section{Glicemia e Lipidemia}

Ao final do estudo, a glicemia de jejum e o colesterol não apresentaram alterações significantes entre os grupos. Já os níveis de triglicérides foram mais elavados nos animais submetidos à dieta hiperlipídica nos grupos SM e SMQ. O uso do flavonóide quercetina não causou alterações significativas nos parâmetros bioquímicos avaliados quando o grupo SMQ foi comparado ao grupo SM, respectivamente, tabela 2 . 
Tabela 1: Consumo alimentar e hídrico, peso corporal e do tecido adiposo retroperitonial e epididimário, nos grupos de estudo

\begin{tabular}{|c|c|c|c|}
\hline & Controle (C) & Síndrome Metabólica (SM) & SM/Quercetina (SMQ) \\
\hline & $\mathrm{N}=12$ & $\mathrm{~N}=12$ & $\mathrm{~N}=12$ \\
\hline \multicolumn{4}{|l|}{ Consumo alimentar } \\
\hline Ingestão diária (g/dia) & $17,0 \pm 2,39$ & $13,1 \pm 2,33 *$ & $13,9 \pm 2,46 *$ \\
\hline Ingestão energética (Kcal/dia) & $63,0 \pm 8,87$ & $68,3 \pm 12,16$ & $72,3 \pm 12,79$ \\
\hline \multicolumn{4}{|l|}{ Consumo hídrico } \\
\hline Ingestão diária (ml/dia) & $22,1 \pm 3,13$ & $16,99 \pm 2,97 *$ & $17,1 \pm 2,74 *$ \\
\hline \multicolumn{4}{|l|}{ Peso corporal (g) } \\
\hline $1^{\mathrm{a}}$ semana & $56,6 \pm 9,27$ & $57,50 \pm 5,36$ & $57,5 \pm 2,74$ \\
\hline $10^{a}$ semana & $258,2 \pm 27,9$ & $303,6 \pm 36,1^{*}$ & $293,7 \pm 29,4^{*}$ \\
\hline $20^{a}$ semana & $317,6 \pm 40,5$ & $412,3 \pm 39,0^{*}$ & $405,1 \pm 39,7^{*}$ \\
\hline Gordura retroperitonial (g) & $5,4 \pm 1,76$ & $14,8 \pm 3,07 *$ & $16,0 \pm 4,23 *$ \\
\hline Gordura epididimária (g) & $2,3 \pm 0,68$ & $6,5 \pm 1,50 *$ & $7,1 \pm 1,58^{*}$ \\
\hline
\end{tabular}

Dados apresentados em média \pm desvio padrão; * $\mathrm{p}<0,05$ vs grupo controle (teste de Dunnet). Os grupos SM e SMQ não são diferentes entre si.

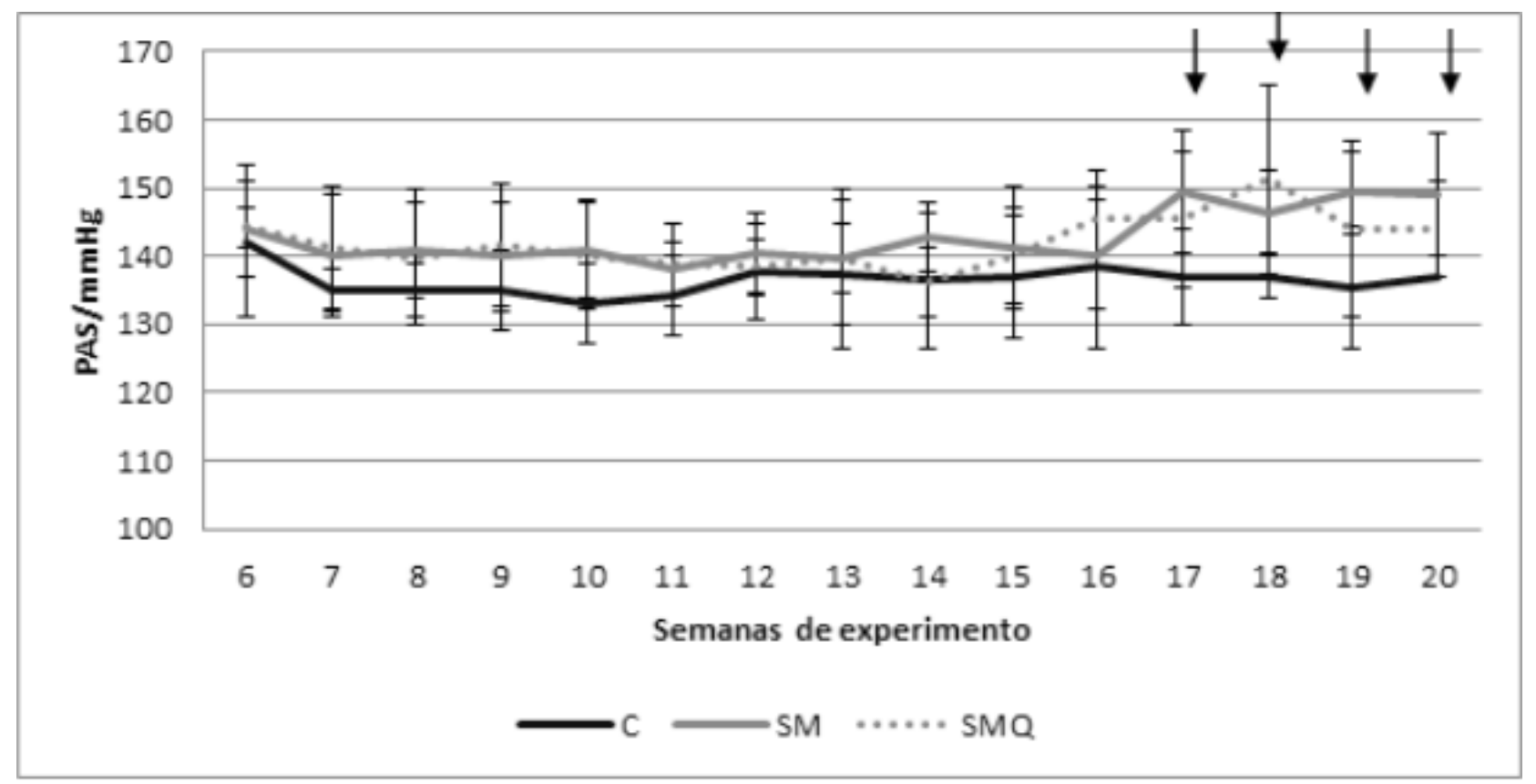

Gráfico 1: Pressão arterial sistólica, por medida indireta, dos grupos estudados. Dados apresentados em média. PAS apresentada como média, da média individual de 10 leituras obtidas. Os grupos foram comparados, entre si, por ANOVA e teste de Dunnet. Seta - p<0,05 grupo SM vs grupo C (teste de Dunnett). Os grupos SM e SMQ não são diferentes entre si.

Tabela 2: Parâmetros bioquímicos nos grupos experimentais ao final de 20 semanas do experimento

\begin{tabular}{lccc}
\hline & Controle $(\mathrm{C})$ & Síndrome Metabólica (SM) & SM/Quercetina (SMQ) \\
\hline & $\mathrm{N}=11$ & $\mathrm{~N}=9$ & $\mathrm{~N}=11$ \\
Glicose $(\mathrm{mg} / \mathrm{dl})$ & $142,9 \pm 13,3$ & $133,1 \pm 20,6$ & $130,4 \pm 27,3$ \\
Colesterol $(\mathrm{mg} / \mathrm{dl})$ & $67,6 \pm 4,69$ & $63,2 \pm 9,2$ & $75,3 \pm 14,7$ \\
Triglicerídeos $(\mathrm{mg} / \mathrm{dl})$ & $28,0 \pm 4,8$ & $53,8 \pm 12,4 *$ & $47,8 \pm 16,6 *$ \\
\hline $\begin{array}{l}\text { Dados apresentados em média } \pm \text { desvio padrão; } * \mathrm{p}<0,05 \text { vs grupo controle (teste de Dunnet). Os grupos SM e SMQ } \\
\text { não são diferentes entre si. }\end{array}$ &
\end{tabular}




\section{DISCUSSÃO}

A síndrome metabólica (SM) é caracterizada pela associação de três ou mais comorbidades que incluem obesidade central, hipertensão arterial, intolerância à glicose, aumento nos níveis de triglicérides e redução nos níveis de colesterol HDL (ALEXANDER et al., 2003; HEART et al., 2004; REILLY et al., 2003). No presente estudo, a administração de dieta rica em lipídios por um período de 20 semanas induziu obesidade central, hipertrigliceridemia e hipertensão arterial em ratos Wistar, achados compatíveis com diagnóstico da SM.

Os animais do grupo SM apresentaram um ganho de peso corporal superior ao grupo $\mathrm{C}$ após 10 semanas de consumo da dieta e no grupo SMQ houve também aumento. A diferença de peso se manteve ao final do experimento. Dado que o ganho de peso corporal, as adiposidades e o consumo de alimentos não diferiram entre os grupos SM e $S M Q$, os resultados mostram que a quercetina não alterou o perfil antropométrico e alimentar dos animais.

Estudo clínico realizado para avaliar os efeitos da suplementação de quercetina $(150 \mathrm{mg} / \mathrm{kg})$ sobre a pressão arterial, metabolismo lipídico, marcadores de estresse oxidativo e perfil nutricional em pacientes com sobrepeso ou obesos demonstrou redução na pressão arterial dos pacientes, mas por outro lado os perfis lipídico e nutricional mantiveram-se inalterados (EGERT et al., 2009). Resultados similares foram obtidos em camundongos C57BL/6] submetidos à dieta hiperlipídica enriquecida com o flavonóide quercetina, dose equivalente a $0,8 \mathrm{mg} / \mathrm{g}$, nos quais o flavonóide não alterou o consumo alimentar e, dessa forma, não foram detectadas alterações no peso e adiposidades em relação ao controle (STEWART et al., 2008). Autores sugerem que pode haver diminuição na concentração plasmática de quercetina com consequente adaptação metabólica, diminuindo o impacto do efeito da quercetina sobre o gasto energético (STEWART et al., 2008).

A administração da dieta hiperlipídica, nesse estudo, associou-se ao desenvolvimento de hipertensão arterial que inferimos estar correlacionado ao acúmulo de gordura abdominal e a distúrbios no metabolismo da glicose, como também foi demonstrado por Morris et al. 2005 e DeMarco et al. 2014, o qual observaram que, independente da ingestão energética excessiva e/ou ganho de peso corporal, o principal fator responsável por alterações dos níveis pressóricos foi o acúmulo de gordura abdominal (MORRIS; VELKOSKA; COLE, 2005; DEMARCO, AROOR, 2014). O acúmulo de gordura abdominal promove à ativação do sistema nervoso simpático e reabsorção tubular de sódio, o que contribui para aumentar a resistência vascular periférica e a pressão arterial (HALL; HALL, 1994).

No presente estudo, o tratamento com a quercetina não preveniu o aumento da pressão arterial sistólica, em um estudo clínico também não foram observados benefícios da quercetina relacionado a disfunções da hipertensão (BRÜLL et al., 2017). Esses dados são discordantes de estudo realizado por Rivera et al.., (2008) em ratos Zucker obesos no qual demonstrou a redução da hipertensão arterial sistólica com a mesma dosagem de quercetina utilizada em nosso estudo (RIVERA et al., 2008).

A discrepância entre o presente estudo e dados apresentados, poderia ser atribuída a diferenças entre espécies, ao tempo de exposição dos animais, à obesidade ou a pouca gravidade e magnitude da hipertensão arterial gerada pelo modelo utilizado no presente trabalho, já que estudos que demonstraram redução na pressão arterial promovida pela quercetina foram realizados em modelos com hipertensão grave, com níveis de pressão sistólica de $200 \mathrm{mmHg}$ (EDWARDS et al., 2007), além da diferença na formulação e administração da quercetina, uma vez que outros estudos demonstraram efeito com administração na dieta e em doses diferentes (JALILI et al., 2006; KUIPERS et al., 2018).

Apesar de no presente trabalho não termos encontrados resultados positivos em relação à quercetina, muitos autores vem demonstrando o mecanismo de ação pelo qual a quercetina poderia apresentar benefícios as comormidades relacionadas à síndrome metabólica. Estudos em animais mostraram que a quercetina pode proteger do ganho de peso corporal induzido por dieta rica em gordura e acúmulo de tecido adiposo (EGERT et al., 2009; RIVERA et al., 2008; STEWART et al., 2008). Moon e colaboradores utilizando o modelo de indução de obsesidade por dieta hiperlipídica, mostrou que a quercetina suprimiu a adipogênese, reduzindo os principais níveis de expressão gênica do fator adipogênico C / EBP a e reduziu a lipogênese, regulando negativamente os níveis gênicos de Síntese de ácidos graxos (fatty acid synthase/FAS) e acetyl-CoA carboxylase (ACC), ambas enzimas relacionadas a obesidade (MOON et al., 2013).

A quercetina também tem efeitos anti-inflamatórios no tecido adiposo. Em um estudo com ratos wistar a quercetina suprimiu a expressão de estresse oxidativo e marcadores inflamatórios, incluindo o fator nuclear kappa $B$ (NF-kB), fator relacionado ao fator nuclear (Nrf-) 2 e heme oxigenase (HO-) 1(PANCHAL; POUDYAL; BROWN, 2012). O mesmo efeito anti-inflamatório foi observado em um estudo utilizando a quercetina na mesma dose $(10 \mathrm{mg} / \mathrm{kg}$ de peso corporal) e via de administração do nosso trabalho, porém em um modelo de rato geneticamente obeso, ratos Zucker, melhorando o estado inflamatório do tecido adiposo visceral, suprimindo a expressão de TNF-a e aumentando os níveis de adiponectina, o que indica a recuperação das funções do tecido adiposo, (RIVERA et al., 2008).

\section{CONCLUSÃO}

A quercetina vem sendo utilizada como um grande potencial no tratamento e atenuação das comorbidades que compõem a síndrome metabólica, principalmente devido a sua ação ainti-inflamatória e antioxidante. 
A administração crônica diária da quercetina em modelo experimental de SM induzida por dieta hiperlipídica não alterou de forma significante o perfil nutricional, metabólico e pressórico dos animais. Porém mais estudos são necessários para compreender a ação da quercetina no modelo e método utilizado, para que possamos entender os mecanismos pelos quais não foram observados resultados positivos.

\section{FONTE DE FINANCIAMENTO}

Rede Mineira de Bioterismo e Rede Mineira de Ensaios Toxicológicos e Farmacológicos de Produtos Terapêuticos.

\section{REFERÊNCIAS}

ALEXANDER, C. M. et al.. Prevalence of Coronary Heart Disease Among NHANES III Participants Age 50 Years and Older. Blood Pressure, v. 52, p. 1210-1214, may. 2003.

BIRT, D. F.; HENDRICH, S.; WANG, W. Dietary agents in cancer prevention: flavonoids and isoflavonoids. Pharmacology \& Therapeutics, v. 90, n. 2-3, p. 157-177, may. 2001.

BROWN, L.; PANCHAL, S. K. Rodent models for metabolic syndrome research. Journal of Biomedicine and Biotechnology, v. 2011, p. 1- 4, dec. 2010.

BRÜLL, V. et al.. Acute intake of quercetin from onion skin extract does not influence postprandial blood pressure and endothelial function in overweight-to-obese adults with hypertension: a randomized, double-blind, placebo-controlled, crossover trial. European Journal of Nutrition, v. 56, n. 3, p. 1347-1357, apr. 2017.

DOURMASHKIN, J. T. et al.. Different forms of obesity as a function of diet composition. International Journal of Obesity, v. 29, n. 11, p. 1368-1378, nov. 2005.

EDWARDS, R. L. et al.. Quercetin Reduces Blood Pressure in Hypertensive Subjects. The Journal of Nutrition, v. 137, n. 11, p. 2405-2411, nov. 2007.

EGERT, S. et al.. Quercetin reduces systolic blood pressure and plasma oxidised low-density lipoprotein concentrations in overweight subjects with a high-cardiovascular disease risk phenotype: a double-blinded, placebo-controlled cross-over study. British Journal of Nutrition, v. 102, n. 7, p. 1065 , oct. 2009.

ENES, C. C.; SLATER, B. Obesidade na adolescência e seus principais fatores determinantes. Revista Brasileira de Epidemiologia, v. 13, n. 1, p. 163-171, mar. 2010.

GÓMEZ-HERNÁNDEZ, A. et al.. Differential Role of Adipose Tissues in Obesity and Related Metabolic and Vascular Complications. International Journa of Endrocrinology, v. 2016, p. 1-14, sep. 2016.
GOULARTE, F. et al.. Effects of exposure to a cafeteria diet during gestation and after weaning on the metabolism and body weight of adult male offspring in rats British. Journal of Nutrition, v. 111, $n$. 8, p. 1499-1506, apr. 2014.

HALL, J. E.; HALL, J. E. Hypertension in Obesity, 1994.

HEART, American et al.. Association Conference on Scientific Issues Related to Definition. p. 433-438, 2004.8006116083.

JALILI, T. et al.. Quercetin-supplemented diets lower blood pressure and attenuate cardiac hypertrophy in rats with aortic constriction. Journal of Cardiovascular Pharmacology, v. 47, n. 4, p. 531541, apr. 2006.

KUIPERS, E. N. et al.. Quercetin lowers plasma triglycerides accompanied by white adipose tissue browning in diet-induced obese mice. International Journal of Molecular Sciences, $v$. 19, n. 6, p. 1-14, jun. 2018.

LIN, X. et al.. Developmental pathways to adiposity begin before birth and are influenced by genotype, prenatal environment and epigenome. BMC Medicine, v. 15, n. 1, p. 50 , mar. 2017.

MACHADO, H. et al. Dissociation of antihypertensive and metabolic response to losartan and spironolactone in experimental rats with metabolic sindrome. Jornal brasileiro de nefrologia, v. 34 , n. 4 , p. 328-36, oct-dec. 2012.

MACHADO, H.; DE OliVEIRA GUERRA, M.; MARIA PETERS, V. Implantação e padronIzação da técnIca de aferição indireta da pressão arterial em ratos WIstar da colônia do biotério do centro de biologia da reprodução (CBR). Revista Interdisciplinar de Estudos Experimentais, v. 2, n. 2, p. 60-61, aug. 2010.

MOON, J. et al.. Antiobesity effects of quercetin-rich onion peel extract on the differentiation of 3T3-L1 preadipocytes and the adipogenesis in high fat-fed rats. Food and Chemical Toxicology, v. 58, p. 347-354, aug. 2013.

MORRIS, M. J.; VELKOSKA, E.; COLE, T. J. Central and peripheral contributions to obesity-associated hypertension: Impact of early overnourishment. Experimental Physiology, v. 90, n. 5, p. 697-702, sep. 2005.

NASCIMENTO, A. F. et al. Misclassification probability as obese or lean in hypercaloric and normocaloric diet. Biological Research, v. 41, n. 3, p. 253-259, apr. 2008.

NG, Marie et al. Global, regional, and national prevalence of overweight and obesity in children and adults during 1980-2013: A systematic analysis for the Global Burden of Disease Study 2013. The Lancet, v. 384, n. 9945, p. 766-81, aug. 2014.

OŻAROWSKI, M. et al. Pharmacological Effect of Quercetin in Hypertension and Its Potential Application in Pregnancy-Induced Hypertension: Review of In Vitro, In Vivo, and Clinical Studies. Evidence-based Complementary \& Alternative Medicine (eCAM), v. 2018, n. 2, p. 1-19, dec. 2018. 
PANCHAL, S. K.; POUDYAL, H.; BROWN, L. Quercetin Ameliorates Cardiovascular, Hepatic, and Metabolic Changes in Diet-Induced Metabolic Syndrome in Rats. Journal of Nutrition, v. 142, n. 6, p. 1026-1032, jun. 2012.

PEREZ-VIZCAINO, F. et al. Antihypertensive effects of the flavonoid quercetin. Pharmacological Reports, v.61. n.1, p. 67-75, jan-feb. 2009.

RAMALHO, R.; GUimarÃES, C. Papel do tecido adiposo e dos macrófagos no estado de inflamação- Implicações Clínicas. Acta Medica Portuguesa, v. 21, n.5, p. 489-496, feb. 2008.

REILLY, J. J. et al. Health consequences of obesity. Arch Dis Childhood, v. 88 , p. 748-752, 2003.

RIVERA, L. et al. Quercetin Ameliorates Metabolic Syndrome and Improves the Inflammatory Status in Obese Zucker Rats. Obesity, v. 16 , n. 9, p. 2081-2087, sep. 2008.

SHEN, C. et al.. High fat plus high salt diet induced metabolic syndrome in wistar rat. American Journal of Hypertension, $\mathrm{v}$. 17, n. 5, p. S220, may. 2004.

SIMMONS, R. K. et al.. The metabolic syndrome: useful concept or clinical tool? Report of a WHO Expert Consultation. Diabetologia, v. 53, n. 4, p. 600-605, apr. 2010.

STEWART, L. K. et al.. Quercetin transiently increases energy expenditure but persistently decreases circulating markers of inflammation in C57BL/6] mice fed a high-fat diet. Metabolism: clinical and experimental, v. 57, n. 7 (Suppl 1), p. S39-46, jul. 2008.

VINCENT, G. D.; ANNAYYA, R. A.; JAMES, R. S. The pathophysiology of hypertension in patients with obesity. Nature Reviews Endocrinology, v. 10, n. 6, p. 364-376, jun. 2014.

YANG, R. et al. Quercetin relieved diabetic neuropathic pain by inhibiting upregulated $\mathrm{P} 2 \mathrm{X} 4$ receptor in dorsal root ganglia. Journal of Cellular Physiology, v. 234, n. 3, p. 2756-2764, apr. 2018. 